\title{
Design and Analysis of a Magnetic Off-axis Rotary Position Sensing Device*
}

\author{
Ádám Bakos, István Gőzse, and Alexandros Soumelidis
}

\begin{abstract}
In the paper design and analysis of an off-axis absolute rotary position sensor setup is described that is intended to use in realizing accurate and reliable absolute angle measurement to be applied in precise control of electric (e.g. vehicle) drives. A solution of using magnetic position sensing elements in combination with two differently magnetized ferromagnetic rings is presented. By assuming a specific setup the algorithm of determining absolute position is given, as well by analyzing the measurement errors, a compensation scheme is elaborated. By using this scheme the linearity error of the sensor setup can significantly be reduced without applying any expensive equipment.
\end{abstract}

\section{INTRODUCTION}

Measuring angle in rotating machines e.g. electric motors occurs frequently in the today practice. Incremental angle measurements can be considered to be part of the everyday practice, and a great variety of sensors - including mechanical, optical, and magnetic ones - are available from several vendors. By incremental sensors also absolute angle measurement can be realized, however it needs setting the initial position that in many cases is not convenient to be performed. The ideal solution is realizing direct absolute angle measurement, which as being a more delicate task requiring expensive sensing devices (e.g. multitrack absolute optical encoder discs), cannot be considered to be a commonly used option. [1]

Magnetic rotary encoders - using the principles of Hallor magnetoresistive effect, and being present in the product variety of several semiconductor companies - offer a lowcost, effective solution of measuring absolute angle of rotating parts in the case if a free shaft end is available (i.e. they realize on-axis sensing devices) [2]. In the opposite case, only an off-axis absolute angle measurement method is applicable, but it cannot directly be realized by using these devices, bringing a new idea is necessary to accomplish this task.

There are only a few solutions on the market which are suitable for off-axis rotary position sensing. The measurement principle of these includes the conventional optical and resolver type methods with all of these wellknown drawbacks, but also includes a magnetic type method, which is more beneficial for the task. A magnetic rotary

*The research has been conducted as part of the project TÁMOP4.2.2.A-11/1/KONV-2012-0012: Basic research for the development of hybrid and electric vehicles.

Ádám Bakos, István Gözse, and Alexandros Soumelidis are with the Systems and Control Laboratory, Institute for Computer Science and Control of the Hungarian Academy of Sciences, Kende str. 13-17, Budapest, Hungary, H-1111

(e-mail: bakos.adam;istvan.gozse;alexandros.soumelidis@sztaki.mta.hu) position sensor is e. g. less sensitive to environmental impacts or vibration, and it can be mounted on an easier way. Their high resolution and precision beside to their low cost is also not a negligible fact.

In this paper a solution of using magnetic position sensing elements in combination with two differently magnetized ferromagnetic rings is presented with the purpose to produce accurate off-axis rotary position sensing. A potential application field of these sensors could be rotor position sensing in high precision electric motor drives, e.g. permanent magnet synchronous motor (PMSM) drives controlled by the principle of sine vector fields.

After introducing the measurement principle (Sect. II), a measurement algorithm is proposed (Sect. III), furthermore an extended analysis of errors and the principles of compensating them is given (Sect. IV), finally a short presentation of experimental testing is described (Sect. V).

\section{MEASUREMENT PRINCIPLE}

High resolution off-axis magnetic position sensors commonly use a specially magnetized ring mounted on the rotating part of the construction, and Hall-elements as sensing devices inside an integrated circuit mounted in front of the ring [3]. The magnet has to be close enough to the chip, so that the Hall-elements can detect magnetic field variation as the magnet rotates. It is common, that the magnets are magnetized in such a way that the magnetic field distribution follows a sinusoidal shape along the curvature of the ring. Within one period of this sine wave there is a pole change from north to south, thus it defines a pole-pair. If the Hall-elements are placed in an appropriate way inside the chip, their outputs produce two sinusoidal waveforms as the magnet ring rotates which are $90^{\circ}$ out of phase. By measuring and processing these signals, the absolute position of the magnet can be determined within the actual pole-pair. The absolute position of the concerned pole directly can be determined only in incremental manner. However, if two magnet rings and two sensors are used with some anisotropy in the magnetic poles, the true absolute rotary position can be determined, within the whole revolution of the magnet rings. This idea will be elaborated as follows.

The measurement principle implies that the length of a pole-pair, hence the period of the sinusoidal magnetic field variation, has to be equal to a fixed value determined by the Hall-sensors' arrangement. It is also true, that the output value of a sensor is, in fact, associated with the length of an arc starting from the center of the current pole-pair, and the current rotation angle value can be calculated knowing the radius of the ring. As the length of the pole-pair is fixed, only 
the number of the pole-pairs can be chosen, the radius of the ring is determined.

Let us consider a sensor corresponding to the properties above, which has digital output and has a fixed output code range, determined by its resolution. This range is linearly scaled to the length of the arc between the sensor center point and the center of the current pole-pair. On the magnet ring, the pole-pairs are continuously following each other, thus the sensor output readout is periodically goes from zero to its maximum value and then starts from zero again as the magnet rotates.

If we are using two identical sensors and two magnet rings with different number of pole-pairs, the arc on the ring with the higher number of pole-pairs (that with the longer radius) is longer than on the other at a given absolute angle. Thus, the output of the two sensors is different. It can be shown, that if the pole-pair numbers on the two rings are relative prime, the same sensor output value-pair is never repeated within one revolution of the magnet rings. Thus, the current arc length measured from the absolute 0 position; hence the absolute angle can be determined unambiguously. As each of the sensors provides absolute position within one pole pair, the problem can be traced back to the determination of the ordinal number of the current pole-pair on one of the rings.

\section{MEASUREMENT ALGORITHM}

As the measurement method have to be used in a control system, an absolute position reconstruction algorithm should be constructed that satisfies some real-time implementation issues. Practically, the algorithm should be able to work on a low-cost and simple microcomputer, e.g. an 8-bit microcontroller.

The explanation of the following symbols is depicted in Fig. 1. Denote the actual sensor readout by $x_{i}$ and $x_{o}$, and the number of pole-pairs by $p p_{i}$ and $p p_{o}$ on the inner and outer ring, respectively. Let $N=2^{b}$ to be the sensor resolution, and $L$ to be the length of the pole-pairs on each ring. Denote the angle from the center of the current pole-pair by $\alpha_{i}$ and $\alpha_{o}$, and the arc length by $l_{i}$ and $l_{o}$ on the inner and outer ring, respectively. Symbols with * superscript denotes absolute values, measured from the predefined absolute zero position.

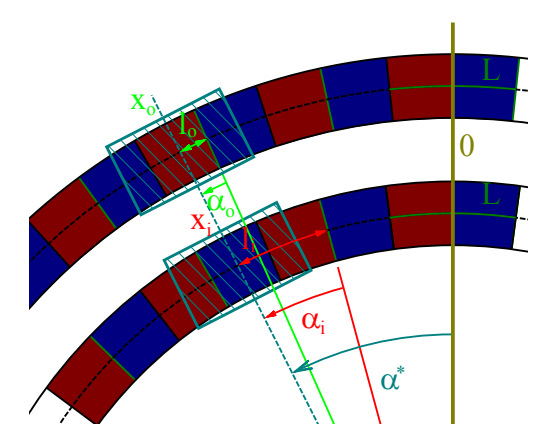

Figure 1. Definition of the used quantities

As the $\left(x_{i}, x_{o}\right)$ sensor output value-pairs are unique within the whole revolution of the rings, it could be an applicable solution to off-line calculate these pairs and store them in a lookup table. Then the on-line algorithm simply searches them form this table. It is obvious, that even if the number of pole-pairs is small, the required memory space and the running time of the searching would be very large, which is impractical.

Another method could be to calculate all the possible absolute angles corresponding to one sensor output, e.g. $\alpha_{i}$ according to the following expression:

$$
\alpha_{i}^{*}[k]=\left(x_{i}+(k-1) p p_{i}\right) / N \cdot L / r_{1}, k=1,2, \ldots, N .
$$

Then calculate all the possible readouts on the other ring corresponding to these absolute angles by the following expression:

$$
X_{o}[k]=\bmod \left(r_{2} \cdot \alpha_{i}^{*}[k] / L \cdot N\right), k=1,2, \ldots, N .
$$

where $\bmod ()$ means modulo division by the $N$ sensor resolution. Equation (2) can be simplified to

$$
X_{o}[k]=\bmod \left(r_{2} / r_{1} \cdot\left(x_{i}+(k-1) N\right)\right), k=1,2, \ldots, N .
$$

The remaining task is to find the actual sensor readout on this ring $\left(x_{o}\right)$ among these $X_{o}[k]$ values. The index $k^{*}$ corresponding to this value identifies the ordinal number of the actual pole-pair on the inner ring, thus the absolute angular position in radians is calculated by

$$
\alpha^{*}=\left(x_{i}+\left(k^{*}-1\right) N\right) /\left(p p_{1} \cdot N\right) \cdot 2 \pi
$$

This method does not require large memory space, but it is requires relatively high amount of calculation.

For real-time applications, a combination of the above two methods is proposed. One sensor output value-pair $\left(x_{i}\right.$, $x_{o}$ ) for each pole-pair on one ring, e.g. on the inner ring has to be calculated off-line and stored in a table. If one of the values in the pair, e.g. $x_{i}$ is the same for all the pairs, than only the $x O$ values must be stored. In this case, the number of the stored values is only $p p_{i}$. For simplicity, the $x_{i}$ values can be those belonging to sensor 0 . The stored $X_{o}[k]$ values are illustrated on Fig. 2.

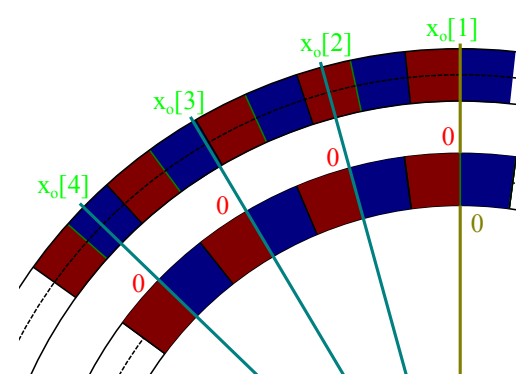

Figure 2. Stored values

The following description is illustrated on Fig. 3. 


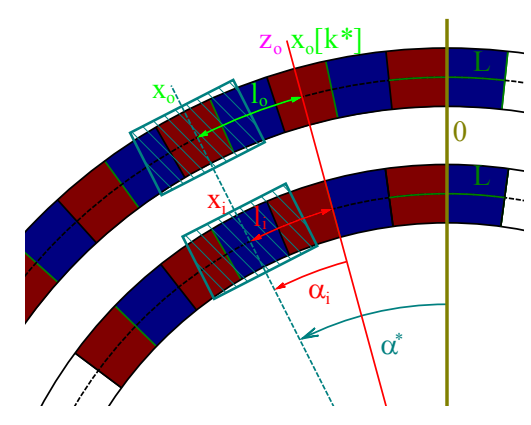

Figure 3. Expanation of the measurement algorithm

Assume that the actual sensor readout is the pair $\left(x_{i}, x_{0}\right)$. In this case $x_{i}$ corresponds to the $l_{i}$ length of the arc measured from the center of the actual pole-pair (with ordinal number yet unknown) on the inner ring. Using the $r_{1}$ radius of the inner ring, the corresponding angle can be calculated:

$$
\alpha_{i}=x_{i} / N \cdot L / r_{1} \text {. }
$$

Then, the arc length $l_{o}$ can be calculated on the outer ring, using the radius $r_{2}$ :

$$
l_{o}=\alpha_{i} \cdot r_{2} .
$$

Subtracting (in terms of modulo) this value from the actual measured $x_{o}$ value gives:

$$
z_{o}=\bmod \left(x_{o}-l_{o} / L \cdot N\right) .
$$

The resulting value corresponds to the $x_{o}$ pair of the previous $x_{i}=0$ value on the inner ring, thus it is located in the stored lookup table.

It is obvious, that the calculation of (7) can be simplified:

$$
z_{o}=\bmod \left(x_{o}-r_{2} / r_{1} \cdot x_{i}\right) .
$$

The remaining task is to find this value in the table. The table index $k^{*}$ of the found value gives the ordinal umber of the actual pole-pair on the inner ring. Then, the absolute angular position can be calculated by

$$
\alpha^{*}=\left(x_{i}+\left(k^{*}-1\right) N\right) /\left(p p_{1} \cdot N\right) \cdot 2 \pi,
$$

which is the same as (4).

It should be noted, since it will be important later, that the values in the stored table are all different, and has a minimal difference between each other, which is determined by the sensor resolution and the number of pole-pairs on the inner ring is $d=N / p p_{i}$.

The benefit of this method is that only one costly calculation, one small-size table and one search on this table is required. This makes it suitable for real-time implementation, even on a simple 8-bit microcontroller.

\section{MEASUREMENT ERRORS}

The algorithm described above, assumes that the all the conditions are ideal. Sensor outputs are deterministic and exactly linearly scaled to the angles. Sensors are mounted perfectly and magnet rings are ideally magnetized. However, in practice neither of the above assumptions is true. This means, that the off-line calculated and stored values are not true for an actual construction and, in worst case, the algorithm can become unusable. According to this, measurement errors have to be analyzed and compensated.

Measurement errors come from different sources. The magnetization of the rings is not perfect and the magnetic field distribution can be deviated from the sinusoidal. Mounting the sensor chip in front of the rings is not exactly precise, i.e. radial displacement of the chip above the magnet can occur. In this case, the length of the arc that is measured by the Hall-sensors is different, i.e. the length of the polepairs is deviated. In practice, there is a requirement for the magnetic field strength too. Naturally, the Hall-elements, the integrated analog front-end and analog-to-digital conversion also suffers from errors. In a practical case, all the above errors are present and combined in a different way. Nevertheless, they can be taken into consideration as linearity error between the true and the indicated position within one pole-pair. The nature of this type of error is illustrated on Fig. 4.

Another type of error, which is related only to the mechanical construction of the measurements system, is the eccentricity. In a practical case, there is a prescribed minimum limit for the width of the magnet rings. If the difference between the radiuses of the two rings is small, e.g. there is only one ring, in fact, with two magnetic tracks, the sensors cannot be mounted in line (collinearly), but have to be shifted in angle. If the magnet rings are mounted eccentrically to the axis of the rotating and this angle shift between the sensors is present, it introduces a linearity error, within one revolution of the rings.

To compensate these errors, further investigation has to be done.

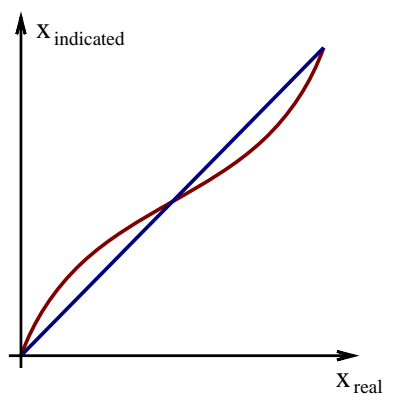

Figure 4. Linearity error

\section{A. Analysis and compensation of eccentricity}

Eccentricity is the one of the main error sources. The reason of this is the angle drift between the sensors what is introduced by the eccentricity. Fig 5 . shows the geometry of an eccentric setup. In the figure point " $\mathrm{C}$ " is the center of the 


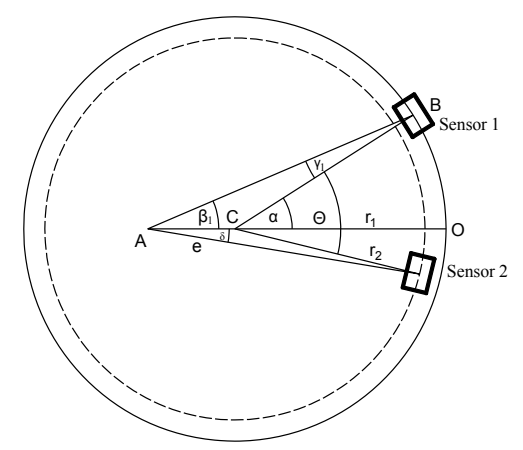

Figure 5. Geometrical setup of the eccentric magnetic ring

real rotation, point " $\mathrm{A}$ " is the center of the magnet ring, so line segment "e" is the eccentricity. To make the discussion easier, the zero angle is assigned to point " $\mathrm{O}$ ", the reason is obvious because that point is collinear with point $\mathrm{A}$ and $\mathrm{B}$, so the eccentricity does not generate error at that point. To evaluate the error introduced by the eccentricity, the difference between the measured and the true rotation angle has to be determined. When the shaft rotates $\alpha$ degree the Sensor 1 is at point $B$ and measures $\beta_{1}$ degree. It is obvious that:

$$
\beta_{1}=\arctan \left(\frac{r_{1} \cdot \sin (\alpha)}{r_{1} \cdot \cos (\alpha)+e}\right),
$$

Sensor 2 measures $\delta$ degree and it is also rotated by $\theta$ degree relative to Sensor 1, but each sensor should measure $\alpha$, so the rotation angle is subtracted from the measurement of Sensor 2. Since there is eccentricity, the Sensor 2 measurement is not exactly $\alpha$ even if the compensation with $\theta$ is done. So Sensor 2 measures:

$$
\beta_{2}=\delta+\Theta=\arctan \left(\frac{r_{2} \cdot \sin (\alpha-\Theta)}{r_{2} \cdot \cos (\alpha-\Theta)+e}\right)+\Theta .
$$

To determine the absolute position, the relative error between the sensors has to be less than the above discussed limit. The relative error introduced by the eccentricity is:

$$
\text { error }=\beta_{1}-\beta_{2} \text {. }
$$

Substituting (10) and (11) into (12) gives the formula for relative error and the maximum of the absolute value of this function can be used to evaluate whether the eccentricity is in conformity with the requirement. Although, this approach answers the question, it does not say too much about the underlying mechanism, so further analytical discussion could be helpful.

It is easy to see, that

$$
\gamma_{1}=\alpha-\beta_{1}, \frac{\sin \left(\alpha-\beta_{1}\right)}{e}=\frac{\sin \left(\beta_{1}\right)}{r_{1}},
$$

thus

$$
\alpha=\arcsin \left(\frac{e}{r_{1}} \cdot \sin \left(\beta_{1}\right)\right)+\beta_{1} .
$$

Furthermore, the same is true for Sensor 2 and $\beta_{2}$.
In (14), the argument of the arcsin function is is small because $\sin (\beta)$ is equal maximum to 1 and the typical value of $e / r_{1}$ is less than one hundreds in practical cases, so:

$$
\alpha=\frac{e}{r_{1}} \cdot \sin \left(\beta_{1}\right)+\beta_{1} .
$$

Further simplification can be made if one considers that $\alpha$ and $\beta_{1}$ is almost the same so sinus $\beta_{1}$ can be substituted by sinus $\alpha$. So $\beta$ can be approximately expressed as:

$$
\beta_{1}=\alpha-\frac{e}{r_{1}} \cdot \sin (\alpha)
$$

According to (16) the measured angle differs from the real angle with a sinusoid, if the $e / r_{1}$ ratio is small. So the error is

$$
\text { error }=\beta_{1}-\beta_{2}=\frac{e}{r_{2}} \cdot \sin (\alpha-\Theta)-\frac{e}{r_{1}} \cdot \sin (\alpha) .
$$

Using this formula the maximum of the error can be determined with basic trigonometric identities:

$$
\max (\text { error })=\sqrt{\left(\frac{e}{r_{2}}\right)^{2}+\left(\frac{e}{r_{1}}\right)^{2}+2 \cdot\left(\frac{e}{r_{1}}\right) \cdot\left(\frac{e}{r_{2}}\right) \cdot \cos (\Theta)} .
$$

Equation (18) can be reformulated:

$$
\max (\text { error })=e \cdot \sqrt{\left(\frac{1}{r_{2}}\right)^{2}+\left(\frac{1}{r_{1}}\right)^{2}+2 \cdot\left(\frac{1}{r_{1}} \cdot \frac{1}{r_{2}}\right) \cdot \cos (\Theta)} .
$$

This means, that the maximum of the error is linear function of the eccentricity and the sensitivity of the error can be easily calculated since:

$$
\frac{\partial \max (\text { error })}{\partial e}=\sqrt{\left(\frac{1}{r_{2}}\right)^{2}+\left(\frac{1}{r_{1}}\right)^{2}+2 \cdot\left(\frac{1}{r_{1}} \cdot \frac{1}{r_{2}}\right) \cdot \cos (\Theta)} .
$$

Consider the following practical case:

$$
r_{1}=14 \mathrm{~mm} \quad r_{2}=12 \mathrm{~mm} \quad \Theta=40^{\circ} \quad e=0.05 \mathrm{~mm} .
$$

The sensitivity of this case is 8.3357 degree $/ \mathrm{mm}$, so the maximum relative error caused by eccentricity is 0.417 degree that is reasonably high while the eccentricity is in the same order of the magnet ring manufacturing precision. So the sensing method could be extremely sensitive to the eccentricity. Easy to see that $r_{1}, r_{2}$ and $\theta$ do not play a significant role in practical cases compared to eccentricity $e$.

To decrease the effect of the eccentricity, two additional sensors can be used arranged opposite to each other. The geometrical setup of one sensor pair is shown in Fig. 6.

The opposite sensors are placed at point B and D. Since the two sensors can not be placed exactly, there is a placement error angle $\varepsilon_{1}$. The placement error angle is originated from the soldering and mounting precision. The sensor at point $\mathrm{B}$ according to (16) approximately measures:

$$
\beta_{1}^{\prime}=\alpha-\frac{e}{r_{1}} \cdot \sin (\alpha) .
$$


And sensor at point $\mathrm{D}$ measures:

$\beta_{1}{ }^{\prime \prime}=\alpha-\frac{e}{r_{1}} \cdot \sin \left(\alpha-\pi-\varepsilon_{1}\right)=\alpha+\frac{e}{r_{1}} \cdot \sin \left(\alpha-\varepsilon_{1}\right)$.

In (22) the possible difference between the radiuses of the two sensors is neglected.

It is easy to see that the average of the two measurements approximate $\alpha$, if angle $\varepsilon_{1}$ is small. If the value of $\varepsilon_{1}$ in (23) is zero, then $\beta_{1}$ become equal to $\alpha$. Of course this holds if the $e / r_{1}$ ratio is small:

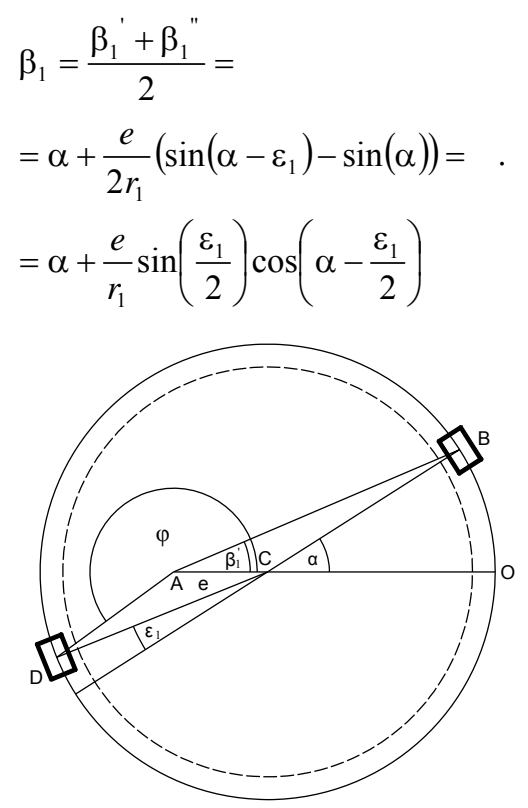

Figure 6. Eccentricity compensation with additional sensors

The same equations can be derived for the other sensor pairs, the only difference is the $\theta$ angle between the sensors as presented above in Fig. 6., thus

$$
\begin{gathered}
\beta_{2}{ }^{\prime}=\alpha-\frac{e}{r_{2}} \cdot \sin (\alpha-\Theta), \\
\beta_{2}{ }^{\prime \prime}=\alpha+\frac{e}{r_{2}} \cdot \sin \left(\alpha-\Theta-\varepsilon_{2}\right) \\
\beta_{2}=\frac{\beta_{2}{ }^{\prime}+\beta_{2}{ }^{\prime \prime}}{2}=\alpha+\frac{e}{r_{2}} \sin \left(\frac{\varepsilon_{2}}{2}\right) \cos \left(\alpha-\Theta-\frac{\varepsilon_{2}}{2}\right) .
\end{gathered}
$$

The error and the sensitivity of the new arrangement can be derived in a similar way that was discussed, so:

$$
\text { error }=\beta_{1}-\beta_{2},
$$

$$
\begin{aligned}
& \max (\text { error })=e \cdot\left(\begin{array}{l}
\left(\frac{1}{r_{1}} \cdot \sin \frac{\varepsilon_{1}}{2}\right)^{2} \cos ^{2}\left(\frac{\varepsilon_{1}}{2}\right)+ \\
+2\left(\frac{1}{r_{1}} \cdot \sin \frac{\varepsilon_{1}}{2}\right)\left(\frac{1}{r_{2}} \cdot \sin \frac{\varepsilon_{2}}{2}\right) \cos \left(\frac{\varepsilon_{1}}{2}\right) \cos \left(\Theta+\frac{\varepsilon_{2}}{2}\right)+ \\
+\left(\frac{1}{r_{2}} \cdot \sin \frac{\varepsilon_{2}}{2}\right)^{2} \cos ^{2}\left(\Theta+\frac{\varepsilon_{2}}{2}\right)+ \\
+\left(\frac{1}{r_{1}} \cdot \sin \frac{\varepsilon_{1}}{2}\right)^{2} \sin ^{2}\left(-\frac{\varepsilon_{1}}{2}\right)++ \\
+2\left(\frac{1}{r_{1}} \cdot \sin \frac{\varepsilon_{1}}{2}\right)\left(\frac{1}{r_{2}} \cdot \sin \frac{\varepsilon_{2}}{2}\right) \sin \left(-\frac{\varepsilon_{1}}{2}\right) \sin \left(-\Theta-\frac{\varepsilon_{2}}{2}\right) \\
+\left(\frac{1}{r_{2}} \cdot \sin \frac{\varepsilon_{2}}{2}\right)^{2} \sin ^{2}\left(-\Theta-\frac{\varepsilon_{2}}{2}\right)
\end{array}\right)^{1 / 2} \\
& \frac{\partial \max (\text { error })}{\partial e}=\left(\begin{array}{l}
\left(\frac{1}{r_{1}} \cdot \sin \frac{\varepsilon_{1}}{2}\right)^{2} \cos ^{2}\left(\frac{\varepsilon_{1}}{2}\right)+ \\
+2\left(\frac{1}{r_{1}} \cdot \sin \frac{\varepsilon_{1}}{2}\right)\left(\frac{1}{r_{2}} \cdot \sin \frac{\varepsilon_{2}}{2}\right) \cos \left(\frac{\varepsilon_{1}}{2}\right) \cos \left(\Theta+\frac{\varepsilon_{2}}{2}\right)+ \\
+\left(\frac{1}{r_{2}}\right)^{2} \cos ^{2}\left(\Theta+\frac{\varepsilon_{2}}{2}\right)+ \\
+\left(\frac{1}{r_{1}} \cdot \frac{\varepsilon_{1}}{2}\right)^{2} \sin ^{2}\left(-\frac{\varepsilon_{1}}{2}\right)+ \\
+2\left(\frac{1}{r_{1}} \cdot \sin \frac{\varepsilon_{1}}{2}\right)\left(\frac{1}{r_{2}} \cdot \sin \frac{\varepsilon_{2}}{2}\right) \sin \left(-\frac{\varepsilon_{1}}{2}\right) \sin \left(-\Theta-\frac{\varepsilon_{2}}{2}\right)+ \\
+\left(\frac{1}{r_{2}} \cdot \sin \frac{\varepsilon_{2}}{2}\right)^{2} \sin ^{2}\left(-\Theta-\frac{\varepsilon_{2}}{2}\right)
\end{array}\right)^{1 / 2}
\end{aligned}
$$

Using the mentioned practical case and if the placement error of the sensor IC-s are about $0.2 \mathrm{~mm}$, then the $\varepsilon_{1}$ and $\varepsilon_{1}$ are about:

$$
\varepsilon_{1}=0,0143 \mathrm{rad} \quad \varepsilon_{2}=0,0167 \mathrm{rad} .
$$

So the maximum error is 0.00325 degree and the sensitivity is 0.0649 degree $/ \mathrm{mm}$. The ratio between the two sensitivities is more than a hundred, which means that the additional sensors make the above mentioned setup feasible, because the IC-mounting precision is better than $0.2 \mathrm{~mm}$. In general case, one should examine the conditions before applying additional sensors.

\section{B. Analysis and compensation of linearity error within a pole-pair}

In ideal conditions the magnetic field of a pole-pair has sinusoid shape and the sensors are placed without error, so its output is proportional to the displacement. There is no doubt that these conditions never occur, hence the function between the displacement and the sensor reading becomes nonlinear. This kind of nonlinearity can be considered to be static nonlinearity, since it depends on the actual absolute position. The aim of the sensor is to measure absolute position, so this kind of nonlinearity should be compensated before the absolute position is determined. This contradiction can be resolved by the assumption that each pole-pair has the same nonlinearity. This assumption can be considered true if the main source of nonlinearity is other than the defective magnetization of the magnet ring, because the other causes can be attributed to geometrical misalignments that are constant during a whole revolution except the eccentricity. To compensate the nonlinearity, one can simply subtract a compensation value from the actual measurement. However, 
determining the compensation values can be challenging, since the angular position should be measured more precisely than that of the actual sensor. The next section describes a method what estimates the nonlinearity without using a more precise angular sensor.

One can assume that if the magnetic ring rotates with a constant angular speed, then the angle measurement is a straight line against time except its nonlinearity that is repeatedly occurs in every whole revolution. As a consequence of the assumption that each pole-pair indicates the same nonlinearity, the nonlinearity can be measured periodically in each pole-pair. The corresponding algorithm is the following.

First step is data acquiring while the magnetic ring is rotating with constant angular speed. To get the nonlinearity, the linear part of the angle measurement should be eliminated; this means linear trend removal. The problem is that removing the linear trend is not ideal, since the angular speed is changing, hence a higher order polynomial trend should be subtracted from the angle measurement.

After the trend is removed, the periodic occurrence of the nonlinearities can become visible. Since only one compensation function can be used, it is a good idea to apply the average of the periods. Since the angular speed is not exactly constant, the periods can have different length, hence they should be resampled using interpolation. Fig. 7. shows the periods that are plotted onto each other.

It is clear that a simple average involves some outliers, which should be avoided. Practically, the main source of outliers is the relatively rapid change in the angular speed, so omitting these periods gives better result. To determine the average without the outliers the following algorithm is used:

1. average every period

2. compare each period with the average

3. if the actual period is "less similar" to the average concerning certain threshold, omit it

4. if there is no omitted period, then finish the algorithm, if there is, start from Step 1 but use only the remaining periods

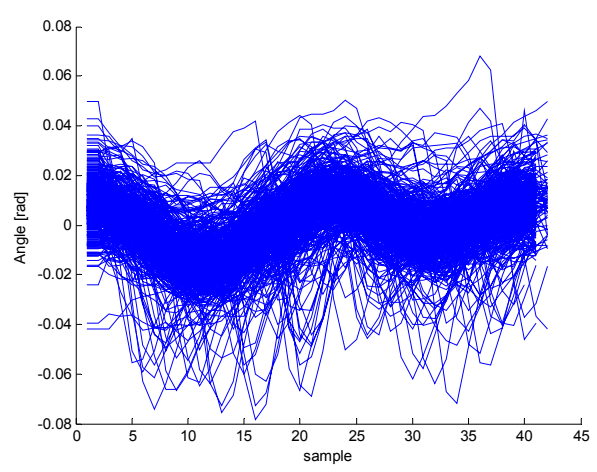

Figure 7. Series of pole-pair nonlinearities

Similarity is determined by

$$
S_{i}=100 \frac{1-\left\|f_{i}-\bar{f}\right\|}{\|\bar{f}-\overline{\bar{f}}\|},
$$

where $\bar{f}$ means the average of the periods, $f_{i}$ means the actual period and $\overline{\bar{f}}$ is the mean value of $\bar{f}$. Fig. 8. shows the result of the averaging algorithm.

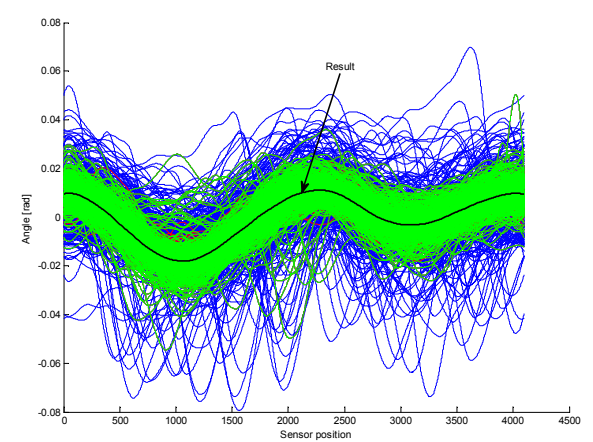

Figure 8. Period selection for average nonlinearity determination

The green periods remained at the end of the algorithm while the others are omitted.

It is very important to validate the result because the algorithm can converge to a bad result.

\section{Modification of the absolute angle calculation algorithm}

To make the absolute angular position calculation algorithm usable in a practical case, some modifications have to be done. The proposed modified algorithm is given as follows.

Three lookup tables must be calculated based upon prior measurements. The rings have to be rotated, sensor values have to be acquired and the following calculations have to be performed.

For the compensation of the linearity error within one pole-pair, the compensation functions for each ring has to be calculated off-line, and stored in two lookup tables. Then, the on-line algorithm has to simply subtract the value addressed from the table by the actual measurement from the measured value.

For the compensation of the linearity error caused by the eccentricity, the $z_{o}$ values have to be calculated based upon the acquired values. Due to the errors, these values will vary from position to position, but spread around an average for each pole-pair. These average values have to be calculated off-line and to be stored in a lookup table. The on-line algorithm has to search for the value in the table that is the nearest to the actual $z_{o}$ value. The $k^{*}$ index of this value is the ordinal number of the actual pole-pair.

As it was mentioned above, the values stored in the lookup table has a minimal difference between each other. The algorithm can only be usable, if the measurement errors 
are less than the half of this difference. In this case, after the search in the table, the value found, nearest to the actual $z_{o}$ value is always correct. In the opposite case, the actual $z_{O}$ value can be close to an incorrect table value, hence the found $k^{*}$ index is erroneous, and the algorithm gives a false result. In Fig. 9.a., a part of the acquired $z_{o}$ values that are used in the off-line table calculation are depicted. In an ideal case, these curves would be straight lines, spaced in equal distances. As it can be seen on the figure, at some rotation angle the curves intersect each other, hence the closest table value could not be found unambiguously. The main source of the depicted effect is the linearity error caused by the eccentricity.

Applying the method that uses two sensors on each ring compensates this error. Thus the algorithm requires further modifications. The two pair of sensors has to be read, and the pairs of values have to be averaged. Then, the off-line table calculation and the on-line reconstruction algorithm have to be evaluated on the basis of these averaged values. A part of the $z_{O}$ values based upon the averaged measurements is depicted on Fig. 9.b. As it can be seen, the curves do not intersect each other, hence the closest value can be found unambiguously, provided that the total measurement error is less than the half of the distance of the two curves.

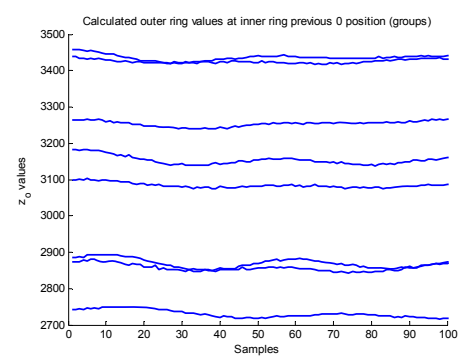

a)

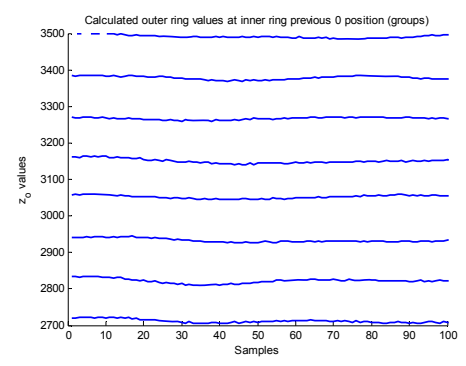

b)

Figure 9. Values for table creation a) uncompensated b) compensated

By using this solution, there are no further excessive and costly requirements for the mechanical construction.

\section{EXPERIMENTAL RESULTS}

The measurement method was developed and tested on a practical construction, which is intended to be used as an electric drive using an outer rotor type motor. The magnetic ring has been designed as a single ferrite ring, with two magnetic tracks. The number of the pole-pair is 37 and 45 on the inner and outer track, respectively. The pole-pair length is $2 \mathrm{~mm}$ on each track and the width of the tracks was selected to be nearly equal. The sensor ICs, which has a resolution of 12-bit has been mounted on a printed circuit board together with an 8-bit microcontroller. There are some mechanical components designed to hold the board and the magnet ring concentrically. The holder of the ring is fixed to the base by bearings and the whole structure is mounted on the fixed shaft of the motor. The holder of the magnet and the rotor are attached by screws. Raw sensor readouts during rotation are depicted on the upper side of Fig. 10. On the middle of the
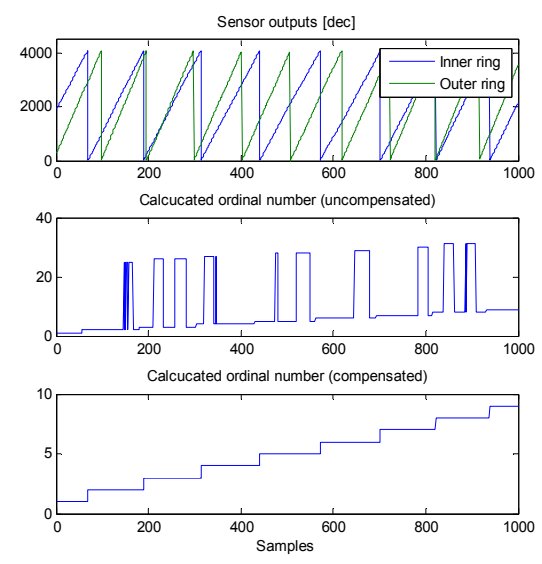

Figure 10. Measurements and reconstructed pole-pair ordinal number

figure and reconstructed pole-pair ordinal number are depicted without linearity and eccentricity compensation. As it can be seen, the results are erroneous. The lower side of Fig. 10. depicts the result using the compensation methods.

\section{CONCLUSION}

Off-axis absolute rotary position sensors play significant role in application fields where accurate and reliable absolute angle measurement is required, e.g. in precisely controlling electric drives associated with electric vehicles. In this paper a solution of using magnetic position sensing elements in combination with two differently magnetized ferromagnetic rings has been presented. By assuming a specific setup the algorithm of determining absolute position has been given, as well by analyzing the measurement errors, a compensation scheme has been elaborated. By using this scheme the linearity error of the sensor setup can significantly be reduced without applying any expensive equipment.

\section{REFERENCES}

[1] R. Crowder, "Electric Drives and Electromechanical Systems" Elsevier, 2006, pp. 107-135.

[2] "Application of Magnetic Position Sensors", Honeywell Solid State Electronics Center, www.magneticsensors.com

[3] Y. Shia, H. Zhanga, X. Jianga, Q. Wenb, B. Hanb "Design of output voltage waveform on magnetic encoder" Journal of Magnetism and Magnetic Materials, Volume 282, November 2004, pp. 317-320. 